\title{
A Novel Approach based on PSO Optimized K-means in MRI Brain Image Segmentation
}

\author{
Abhisha Mano, Assistant Professor, Rajas International Institute of Technology for Women, \\ Nagercoil.
}

\begin{abstract}
Image segmentation is an important task in medical field. It is very much essential to propose a method to analyse the MRI brain image which ease the work of clinicians. In this paper a novel method is proposed to segment MRI brain image into Gray matter(GM), White matter(WM), cerebrospinal fluid(CSF), and skull. Here Particle Swarm Optimization(PSO) is used to optimize the centroid values. These optimized cluster centers are utilized in K-means algorithm to segment the image into various regions. Performance measures such as MSE, PSNR, Dice Coefficient, SSIM is analysed for various regions. The proposed method executes faster than existing method.
\end{abstract}

Keywords: K-means, Particle Swarm Optimization (PSO), Gray matter (GM), White Matter (WM), Cerebrospinal fluid (CSF), GLCM.

\section{INTRODUCTION}

Segmentation plays a major role in medical image processing to segment images into various regions to diagnose the diseases [1]. The images are segmented like bone, muscles and blood vessels, various organs, brain regions etc [2]. Brain image segmentation is very much essential inorder to analyse the tumor in the brain. Many segmentation methods like thresholding, region-growing and clustering are used for MRI brain image segmentation. There are edge based and region based segmentation techniques. MRI brain image has many regions like Gray matter, White matter, CSF, skull etc. An efficient segmentation of brain regions must be done which make clinicians, to provide the appropriate treatment. Sayali D. Gahukar et al., [2] done a work on Fuzzy C-Means along with Ant colony optimization in segmentation of MRI brain image. It works efficiently even if the timing requirement of ACO algorithm is more. Incorporation of intensity inhomogeneity to FCM based on probabilistic approach is shown by Adhikari [3]. The intensity inhomogeneity is obtained using Gaussian method and classified using FCM. Shen, [4] introduced two parameters in segmentation which can handle attraction of neighbours. One is the feature alteration between adjacent pixels in the image. Other was the comparative position of the neighbouring pixels. The parameters are computed using an optimized artificial neural network. Ivana Despotovi [6] presented a new Fuzzy C-Mean for incorporating the spatial data of local image structures into similarity and the membership function to depending on phase congruency features .This conserves homogeneity and is very robust. Archana Lala et al., [7]proposed, Kmeans to partition objects into clusters. Fuzzy c-means, is used in combination with K-means, whose centroid is average of every points. Vishnumurthy et al., [5] presented a technique for segmenting brain MRI using morphological techniques and the outcomes are equated against Maximum expectation technique and Fuzzy C-Means and various performance measures are analysed along with processing time. Abhisha Mano [12][15] and Anand et al[14][13] proposed an efficient approach to enhance the contrast and obtain segmentations of retinal, mammograms, DNA fragments, brain etc.

In this paper, PSO optimized K-means was implemented to extract the regions of brain like Gray matter, White matter, CSF and Skull. The performance measures are analysed using various metrics. 


\section{METHODOLOGY}

The input brain image is taken from Brain Web database. The pre-processing is the process of altering an picked up image into a suitable format [8]. In this pre-processing step, changing the size of the image was done depending on the application. Figure 1 shows the block diagram of proposed method.

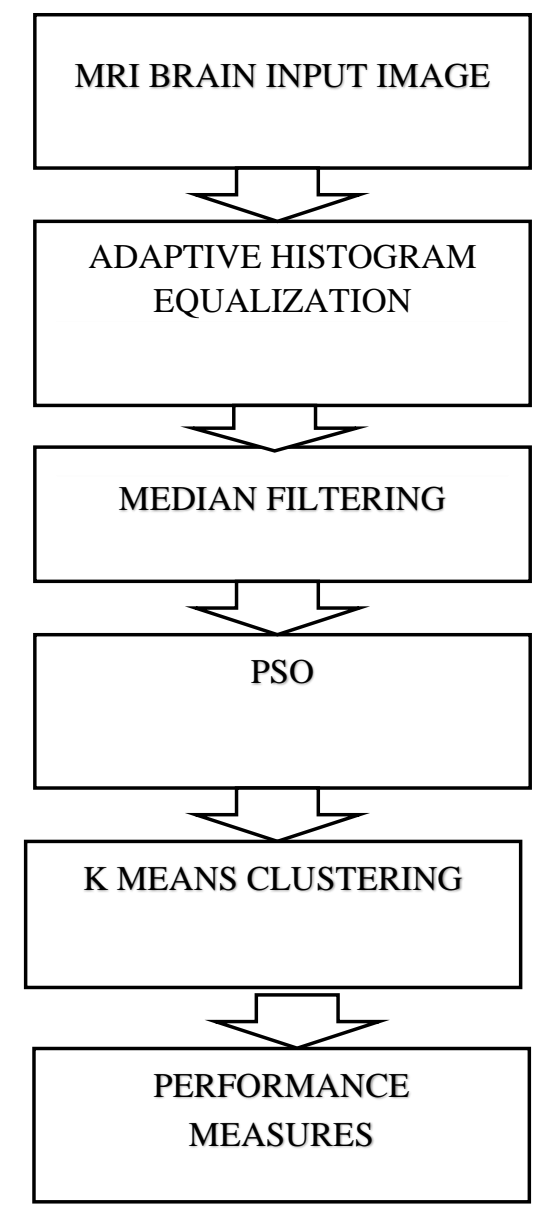

Figure: 1 Proposed System

\subsection{Image enhancement:}

An adaptive histogram equalization is an approach, done inorder to advance the contrast of the image. Here many histograms are calculated, inorder to change the precision value of the image.

\subsection{Filtering:}

A median filter removes the noise, thus providing a smoothing effect and hence preserves the edges of the image. 


\subsection{Particle Swarm Optimization (PSO):}

The Particle Swarm Optimization is a technique which is used to optimize by interative manner to find out the solutions. It is a metaheuristic approach

\section{Algorithm:}

1)For each and every particle initialise the position.

2)The best position is initialised to the initial position.

3)The best known position of swarm is updated.

4)The particle velocity is updated till convergence.

5)When convergence is met, for each and every particle, update the values of particles and swarm's best position.

\subsection{K-means clustering algorithm:}

$\mathrm{K}$-means is a technique which is used to group into clusters depending on the type of the image [8][9]. In K-means, ' $\mathrm{K}$ ' centers are chosen in a way such that one centre for each cluster. The objective function is given by,

$$
\mathrm{J}=\sum_{k=1}^{m} \sum_{i=1}^{n}\left\|y_{i}^{(j)}-c_{k}\right\|^{2}
$$

Where, $\left\|y_{i}{ }^{(j)}-c_{k}\right\|^{2}$ gives the Euclidean distance among $\mathrm{y} i$ and $c_{k}$. The value of $\mathrm{n}$ and $\mathrm{m}$ are number of data points and number of cluster centers respectively.

Steps to be followed:.

Step1: Select the cluster centers in a random manner.

Step2: The distance between each and every data point and cluster centers is calculated.

Step3: The datapoint is allocated to the cluster center which has minimum distance.

Step4: The new cluster center is recalculated using

$$
M_{j}=\frac{1}{n} \sum_{j=1}^{n} y_{i}
$$

Step5: The distance measure is also reallocated.

Step6: If further there was no data point for reallocation then stop. Else repeat from step 3.

\section{RESULTS AND DISCUSSION}

The MRI brain images are chosen from brain web database. The input image was resized to $256 \times 256$. Image enhancement was done to enhance the contrast of the image. Here adaptive histogram equalization was done and it is shown in Fig 2(b). A median filter is used to filter the image. Median filter is more robust and preserves sharp edges [10]. Particle Swarm Optimization is used to optimize the cluster value and it is given to the $\mathrm{k}$ means clustering. Finally four regions such as GM, WM, CSF and skull regions are segmented as shown in Fig 2 d, e, c, f respectively. Table 1 provides the performance measures done on various regions like MSE, PSNR, SSIM, dice coefficient. 


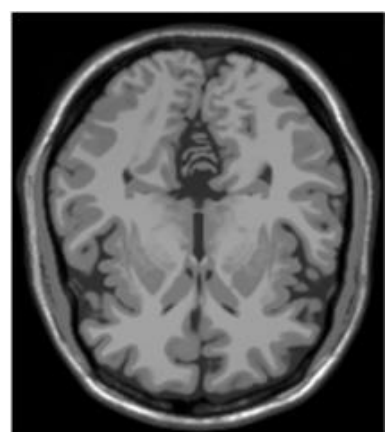

a) Input Image

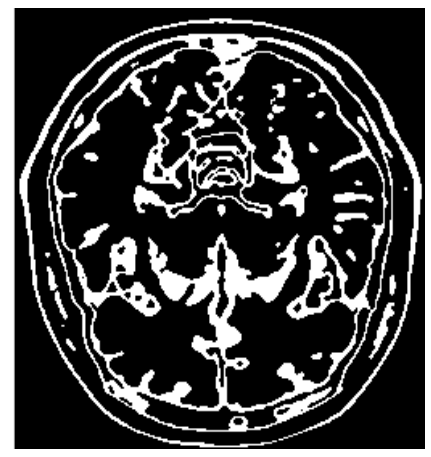

c) $\mathrm{CSF}$

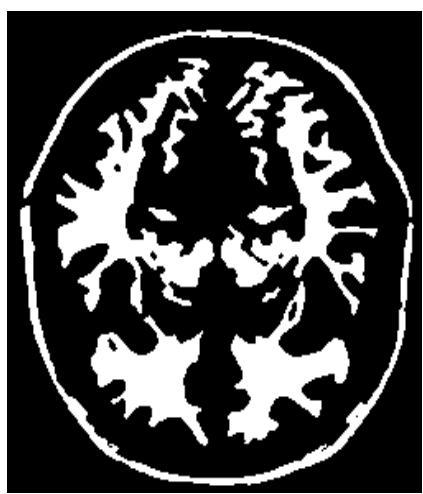

e) WM

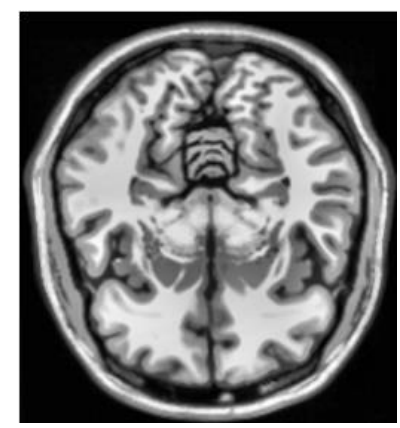

b) Enhanced Image

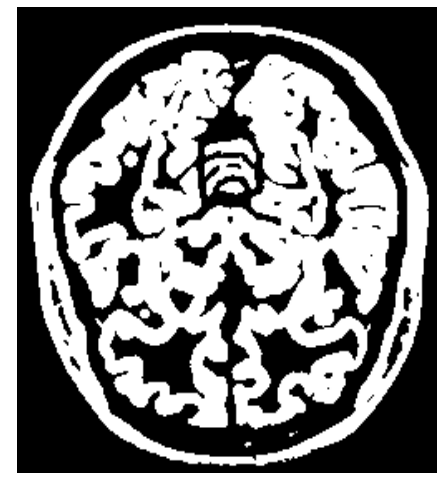

d) GM

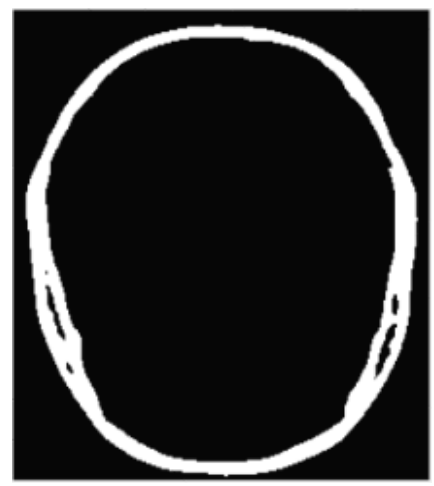

f) skull

Figure 2:Input and segmented regions 
Table 1. Performance Measures

\begin{tabular}{|c|c|c|c|c|}
\hline $\begin{array}{c}\text { Performance } \\
\text { measures }\end{array}$ & $\begin{array}{c}\text { White } \\
\text { Matter }\end{array}$ & $\begin{array}{c}\text { Gray } \\
\text { Matter }\end{array}$ & $\begin{array}{c}\text { Cerebro } \\
\text { Spinal } \\
\text { Fluid }\end{array}$ & Skull \\
\hline $\begin{array}{c}\text { Peak Signal } \\
\text { To Noise } \\
\text { Ratio }\end{array}$ & 6.0723 & 4.1918 & 11.8833 & 8.6134 \\
\hline $\begin{array}{c}\text { Mean Square } \\
\text { Error }\end{array}$ & 0.2469 & 0.3809 & 0.0648 & 0.1376 \\
\hline $\begin{array}{c}\text { Structural } \\
\text { Similarity } \\
\text { Index Map }\end{array}$ & 0.5298 & 0.2431 & 0.5293 & 0.7372 \\
\hline $\begin{array}{c}\text { Dice } \\
\text { Coefficient }\end{array}$ & 0.7378 & 0.4426 & 0.0317 & 0.0604 \\
\hline
\end{tabular}

Table 2 Computation time in sec

\begin{tabular}{|c|c|}
\hline Method & Time(sec) \\
\hline FCM & 8.50 \\
\hline K-means & 7 \\
\hline Proposed method & 6.5 \\
\hline
\end{tabular}




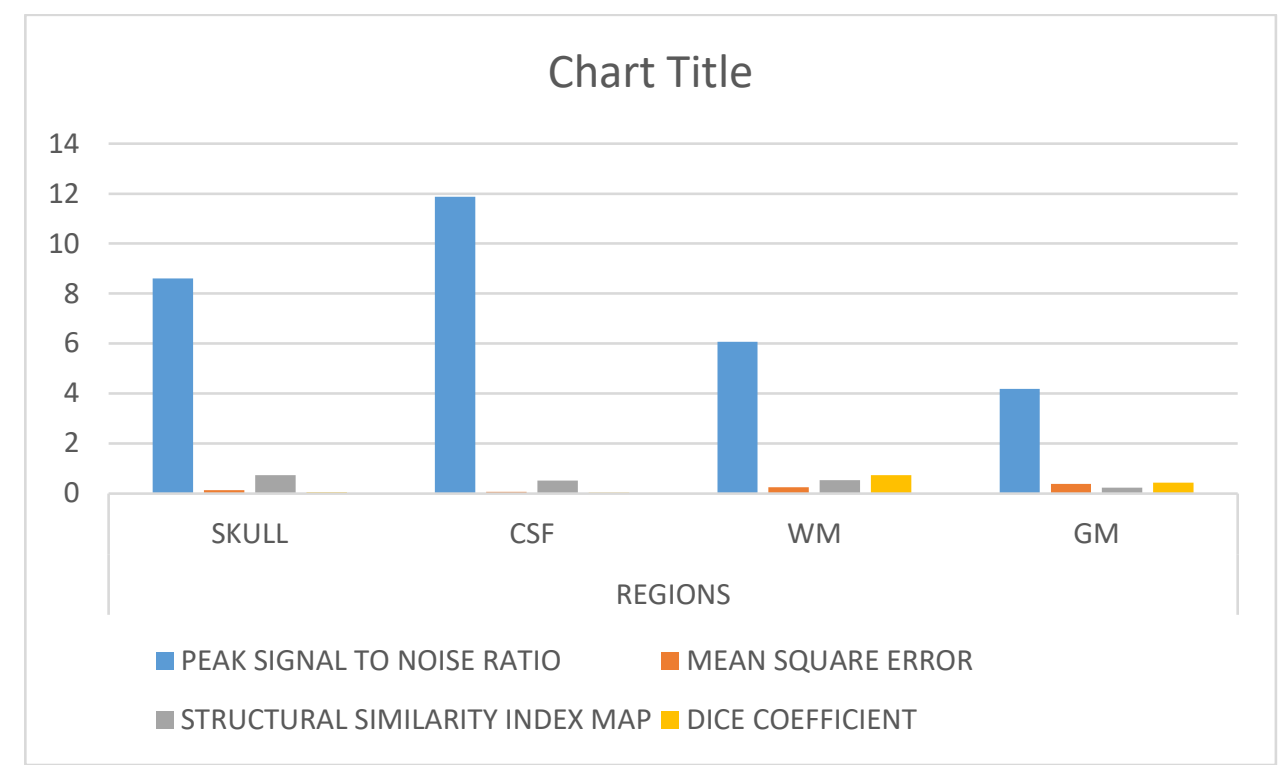

Fig 3. Performance chart

The following parameters were analysed

(i) Mean square Error (MSE):

Mean Square Error gives the quantity of signal fidelity or image fidelity.

(ii) Peak Signal to Noise Ratio (PSNR):

Peak Signal to Noise Ratio helps to analyse how superior the reconstructed image.

(iii) Dice coefficient:

Dice Coefficient shows how two images are similar to each other.

(iv) Structural Similarity Index Map (SSIM):

Structural Similarity Index Map directs the degradation in the quality of image by means of data compression or losses in data broadcast.

Table 2 shows the time taken for execution in seconds. From Table 2 it is understood that the proposed method executes faster compared to existing methods. Fig 3 shows the pictorial representation of performance of various regions.

\section{CONCLUSION}

In this paper, an optimization based on PSO and K-means for separating Gray matter (GM), White matter (WM), Cerebrospinal fluid (CSF) and Skull. The features of these regions were extracted using GLCM. Various performance measures are analysed. The proposed method completes execution in lesser time compared with the existing methods.

\section{REFERENCES}

[1] Zhang, N., Ruan, S., Lebonvallet, S., Liao, Q., \& Zhu, Y. (2011). Kernel feature selection to fuse multi-spectral MRI images for brain tumor segmentation. Computer Vision and Image Understanding, 115(2), 256-269. 
[2] Gahukar, S. D., \& Salankar, S. S. (2014). Segmentation of MRI brain image using fuzzy c means for brain tumor diagnosis. International Journal of Engineering Research and Applications, 4(4), 107-111.

[3]. Adhikari, S. K., Sing, J. K., Basu, D. K., \& Nasipuri, M. (2015). Conditional spatial fuzzy Cmeans clustering algorithm for segmentation of MRI images. Applied soft computing, 34, 758-769.

[4] Shen, S., Sandham, W., Granat, M., \& Sterr, A. (2005). MRI fuzzy segmentation of brain tissue using neighborhood attraction with neural-network optimization. IEEE transactions on information technology in biomedicine, 9(3), 459-467.

[5] Vishnumurthy, T. D., Mohana, H. S., \& Meshram, V. A. (2016, December). Automatic segmentation of brain MRI images and tumor detection using morphological techniques. In 2016 international conference on electrical, electronics, communication, computer and optimization techniques (ICEECCOT) (pp. 6-11). IEEE.

[6] Despotovic, I., Vansteenkiste, E., \& Philips, W. (2013). Spatially coherent fuzzy clustering for accurate and noise-robust image segmentation. IEEE Signal Processing Letters, 20(4), 295-298.

[7] Lala, A., Gupta, J. K., \& Shringirishi, M. (2013). Implementation on K-means Clustering and Fuzzy C-means Algorithm For Brain Tumor Segmentation. International Journal of Computer Engineering \& Science, 3(1), 27-33.

[8] Deepa, V., Benson, C. C., \& Lajish, V. L. (2015). Gray matter and white matter segmentation from MRI brain images using clustering methods. International Research Journal of Engineering and Technology (IRJET), 2(8), 913-921.

[9] Dhanalakshmi, P., \& Kanimozhi, T. (2013). Automatic segmentation of brain tumor using KMeans clustering and its area calculation. International Journal of advanced electrical and Electronics Engineering, 2(2), 130-134.

[10] Selvaraj, D., \& Dhanasekaran, R. (2013). MRI brain image segmentation techniques-A review. Indian Journal of Computer Science and Engineering, 4(5), 364-381.

[11] Kaur, J., \& Gupta, P. (2012). Fuzzy logic based adaptive noise filter for real time image processing applications. International Journal of Computer Science Issues (IJCSI), 9(4), 269.

[12] Mano, Abhisha (2020): CONTRAST ENHANCED SUPERPIXEL BASED SEGMENTATION OF RETINAL IMAGES. TechRxiv. Preprint. https://doi.org/10.36227/techrxiv.12254240.v1.2020

[13] Anand, S., Lakshmanan, B., Murugachandravel, J., Valarmathi, K., Mano, A., \& Kavitha, N. (2019).Wavelet-Based Automated DNA Sizing of Fragments through AFM Image Processing. International Journal of Engineering and Advanced Technology.8(5) .231-238.

[14] Anand, S., Murugachandravel, J., Valarmathi, K., Abhisha Mano \& Kavitha,N.(2019). Contrast Enhancement of Mammograms and Microcalcification Detection. International Journal of Recent Technology and Engineering (IJRTE) ,8(4).3926-3932.

[15] Abhisha Mano \& Anand, S.(2019). GSO based weight Optimized Artificial Neural Network with Modified Active Contour for Brain Tumor Segmentation. Tierärztliche Praxis,39(11).184204. 
